\title{
Effects of Variable Fluid Properties on Double Diffusive Mixed Convection with Chemical Reaction Over an Accelerating Surface
}

\author{
Girinath Reddy Mottupalle 1**(D), Dinesh Pobbathy Ashwathnarayana 1(D), Basavaraj M Shankarappa 1(D), \\ Aruna Agrahara Sanjeevamurthy 1 (D)
}

1 Department of Mathematics, M. S. Ramaiah Institute of Technology (Affiliated to VTU), Bangalore -560054, Karnataka, India.; mgrmathes@gmail.com (G.R.M); dineshdpa@msrit.edu (D.P.A.); basavarajms149@gmail.com (B.M.S.); aruna0as@gmail.com (A.A.S.);

* Correspondence: mgrmathes@gmail.com (G.R.M);

Scopus Author ID 57215905684

Received: 26.07.2021; Revised: 5.09.2021; Accepted: 9.09.2021; Published: 17.10.2021

\begin{abstract}
In this study, we investigate the effect of variable fluid properties such as variable viscosity, porosity, permeability, thermal conductivity, and solutal diffusivity on double-diffusive mixed convective flow over an accelerating surface under the influence of a higher-order chemical reaction. The governing equations of the physical problem involve a coupled nonlinear partial differential equations and which are transformed into a coupled nonlinear ordinary differential equations using a suitable similarity transformation. Numerical computation using shooting technique is adopted to study the physical characteristics of velocity, temperature and concentration for various values of non-dimensional parameters like Prandtl number, Eckert number, buoyancy parameters, viscosity parameter, porous parameter, a ratio of thermal conductivities, a ratio of solutal diffusivities and chemical reaction parameter etc are involved in the problem. The computed numerical results are presented in the graphs to illustrate the details of the flow characteristics and their dependence on physical parameters. Our computed results are compared with earlier works of Seddeek in the absence of a magnetic field and found in good agreement.
\end{abstract}

Keywords: variable fluid properties; double-diffusion; mixed convection; accelerating surface; chemical reaction.

(C) 2021 by the authors. This article is an open-access article distributed under the terms and conditions of the Creative Commons Attribution (CC BY) license (https://creativecommons.org/licenses/by/4.0/).

\section{Nomenclature:}

Prandtl number: $\quad \operatorname{Pr}=\frac{\rho v_{\infty} C_{p}}{k}$

Eckert number: $\quad E=\frac{b^{2} x^{2}}{C_{p}\left(T_{w}-T_{\infty}\right)}$

Schmidt number: $S c=\frac{v_{\infty}}{D_{m}}$

Reynolds number: $\quad R e=\frac{u_{0} x}{v}$

Ratio of viscosities: $\quad \alpha^{*}=\frac{\mu}{\bar{\mu}}$

Porous parameter: $\quad \sigma=\frac{v}{b k_{0}}$
Local temperature Grashof number: $G r_{t}=\frac{g \beta\left(T_{w}-T_{\infty}\right) x^{3}}{v_{\infty}{ }^{2}}$

Local mass Grashof number: $\quad G r_{m}=\frac{g \beta^{*}\left(C_{w}-C_{\infty}\right) x^{3}}{v_{\infty}{ }^{2}}$

Chemical reaction parameter: $\quad K_{r}=\frac{K_{R}\left(C_{w}-C_{\infty}\right)^{l-1} v}{b D_{m}}$

Constant injection: $\quad V_{w}=m(b v)^{\frac{1}{2}}$

Viscosity parameter (PSTC) : $\theta_{r}=\frac{T_{r}-T_{\infty}}{T_{w}-T_{\infty}}=-\frac{1}{\gamma\left(T_{w}-T_{\infty}\right)}$

Viscosity parameter (PWHCF) $: g_{r}=\frac{T_{r}-T_{\infty}}{T_{w}-T_{\infty}}=-\frac{1}{\gamma\left(T_{w}-T_{\infty}\right)}$ 
Mass buoyancy parameter: $G c=\frac{G r_{m}}{\mathrm{Re}^{2}}$

$f$ : Non-dimensionless stream function

$\sigma^{*} \quad$ : Ratio of thermal conductivities

$\gamma^{*} \quad$ : Ratio of solutal diffusivities
Temperature buoyancy parameter: $G s=\frac{G r_{t}}{\operatorname{Re}^{2}}$

$\theta(\eta):$ Dimensionless temperature in PSTC case

$g(\eta)$ : Dimensionless temperature in PWHCF case

$H(\eta)$ : Dimensionless concentration in PSTC case

$h(\eta) \quad$ : Dimensionless concentration in PWHCF case

\section{Introduction}

During the last few decades, a study of fluid flow past an infinite vertical heated porous plate, stretching sheet, horizontal flat plate with/without accelerating surface is of very significant importance due to its wide range of applications in much industrial processing such as coating and polymer processing, paper production, food processing, glass-fiber production, and hot rolling. However, the problem of combined heat and mass transfer flow of a laminar incompressible boundary layer over an accelerating or moving surface embedded in a saturated porous medium has a remarkable application in the fields of chemical engineering and metallurgy. Also, most of the earlier studies on double-diffusive convection through porous media with constant fluid properties or with varying one or two variable fluid properties like variable viscosity, porosity, permeability, etc., have been considered in the literature, and it has a wide range of practical and industrial applications such as grain storage, groundwater contamination, chemical transport in packed bed reactors, disposal of waste material and many other. Most of the above practical applications are related to the existing studies in the literature based on the constant physical properties of the fluid flow.

Lai and Kulacki [1] investigated the effect of variable viscosity on convective heat transfer flow through a saturated porous medium over a porous vertical flat surface. Acharya et al. [2] studied the effect of heat source on double-diffusive convective flow over an accelerating surface under the influence of suction and blowing. Seddeek [3] examined the combined effects of variable viscosity and radiation on an unsteady MHD convective flow over a continuously moving boundary through a porous medium. Chandrasekhar and Namboodiri [4] explained the effect of variable permeability on velocity and temperature distributions through a porous medium. Later, the effects of Soret and Dufour on double-diffusive mixed convective flow with suction and blowing over an accelerating flat surface in the presence of variable viscosity and heat source were carried out by Seddeek [5]. Further, Nalinakshi et al. [6] gave the numerical approach to studying fluid properties' effects on the double-diffusive mixed convective flow. Furthermore, Girinath Reddy et al. [7-8] presented Soret and Dufour's effects on mixed double-diffusive convective flow in the presence of variable viscosity and porosity over an accelerating surface with and without internal heat generation.

Krishna Prasad et al. [9] studied the Soret effect on unsteady mixed MHD convection flow over an accelerated wavy vertical plate through an embedded Darcian porous medium. Manjunatha et al. [10] demonstrated the heat transfer of MHD free convective hybrid nanofluids with the basic properties of fluids. Shilpa et al. [11] gave the analytical approach for the mixed convective flow of Casson fluid through a Porous channel. Sravan Kumar et al. [12] theoretically analyzed MHD free convective nanofluid flow characteristics over an exponentially accelerating vertical plate. Further, Khan et al. [13] described the effects of variable thermal features on the unsteady triple diffusive flow of Eyring-Powell nanofluid over a periodically accelerating surface. Ayegbusi Florence et al. [14] mentioned the effects of thermophoresis and thermal radiation on an 
unsteady MHD heat and mass transfer along with a moveable vertical plate. Ramachandramurthy et al. [15] investigated theoretically the effects of variable viscosity and internal heat source on linear and weakly nonlinear Rayleigh-Bénard convection with rotation. Basavaraj et al. [16] explained a nonlinear MHD oscillatory mixed convective flow through a semi-infinite vertical embedded porous plate.

Nima et al. [17] demonstrated the effects of variable porosity on double-diffusive bioconvective flow with motile microorganisms through a vertical saturated porous material. Anantha Kumar et al. [18] examined the MHD convective heat transfer characteristics on micropolar stagnation point fluid flow past an exponentially curved surface. Wahab [19] et al. explained thermal conductivity and temperature-dependent viscosity effects on MHD nonNewtonian fluid flow over a nonlinearly stretching sheet. Further, Nalinakshi et al. [20] analyzed the combined effects of thermo-diffusion and diffusion-thermo on the MHD Forchheimer model over a heated vertical plate. Sami Ullah Khan et al. [21] introduced the effects of variable thermal conductivity on electrical MHD Carreau nanofluid over oscillatory stretching surface through a porous medium. Gangadhar et al. [22] reported the variations of suction/injection on boundary layer flow of nanofluids with heat absorption/generation over a stretching sheet under the influence of viscous dissipation. Uma et al. [ 23] studied the Forchheimer effect on mixed convective MHD dusty viscoelastic Couette flow with Soret and Dufour effects in an irregular channel.

The combined effects of heat and mass transfer or mass transfer along with chemical reaction on a laminar incompressible flow along with the porous plate with/without accelerating surface in the presence of one or two variable fluid properties involved in many applications in the field of the chemical engineering process, geothermal reservoirs and petroleum industries and which has been studied by many authors in the previous studies. The chemical reaction effect varies concerning its order and the type of chemical reaction (heterogeneous or homogeneous). Apelblat [24] introduced the analytical solutions for the problem of mass transfer of the fluid flow under the influence of first-order chemical reactions subjected to different domains. Afify and Ahmed [25] explained the heat and mass transfer by natural convection about a stretching surface in the presence of a chemical reaction. Rashad et al. [26] studied the mixed convection boundary layer over a stretching sheet which is embedded in a porous medium with chemically reactive species by numerical method.

Shreedhar et al. [27] considered the effect of chemical reaction on an unsteady MHD laminar two-dimensional natural convective flow past a vertical plate through a porous medium with heat absorption. Musa Antidius Mjankwi et al. [28] demonstrated the chemical reaction and thermal radiation effects on an unsteady magnetohydrodynamics flow of nanofluid over an inclined stretching sheet with variable fluid properties. Prasad et al. [29] explained mathematically to analyze the effect of chemical reaction on magnetohydrodynamic Casson fluid flow with a nonuniform heat source or sink across a slender sheet. Dinesh et al. [30] attempted to study the combined effects of Forchheimer and radiation absorption for an unsteady magnetohydrodynamic dusty viscoelastic Couette flow in an irregular channel in the presence of chemical reaction. Shafiq et al. [31] investigated the double-diffusive convective flow of motile microorganisms' secondgrade nanofluid. Sehra et al. [32] studied an incompressible Newtonian unsteady MHD viscous fluid flow for heat and mass transfer by natural convection with time-dependent arbitrary shear stresses over an infinite vertical plate under chemical molecular diffusivity.

Hence, the main objective of this research work is to study and analyze the higher-order chemical reaction effect of double-diffusive mixed convective flow over an accelerating surface 
with variable fluid properties such as variable viscosity, porosity, permeability, thermal conductivity, and solutal diffusivity.

\section{Materials and Methods}

\subsection{Mathematical formulation of the problem.}

A two-dimensional $(x \& y)$, double-diffusive (heat and mass transfer), mixed (free-forced) convective flow of an incompressible, viscous, steady (independent of time), and laminar fluid over an accelerating vertical porous flat plate embedded saturated through a porous medium is considered. Here, the variable fluid properties like variable viscosity, porosity, permeability, thermal conductivity, and solutal diffusivity under the influence of higher-order chemical reaction and all other fluid properties are isotropic and constant. The vertical coordinate axis represents as $x$-axis, which runs along with the accelerating vertical plate, $y$-axis is perpendicular to it, and acceleration due to gravity plays in the opposite direction to the $x$-axis. Due to accelerating surface, the linear velocity $u=b x$ is maintained along $x$-axis and the constant injection velocity $v=v_{w}$ is maintained along $y$-direction. The coordinate system and the physical model of the fluid flow are shown in Figure 1.

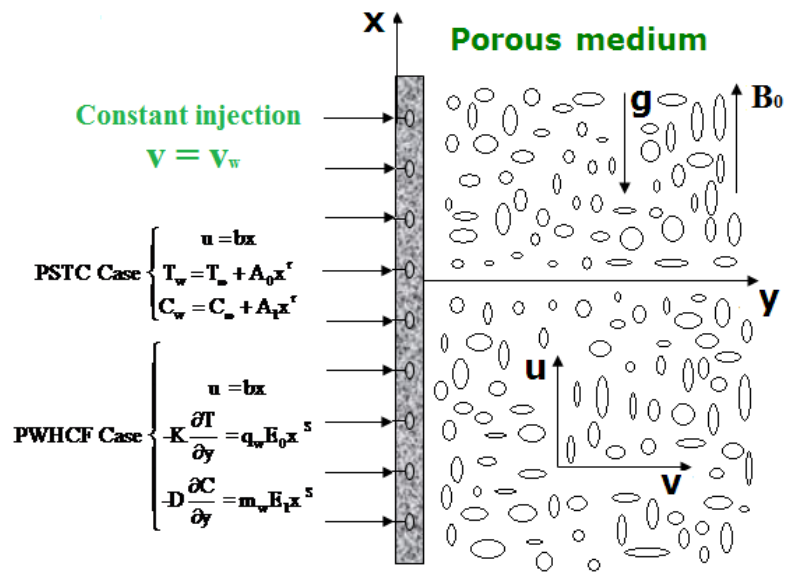

Figure 1. Physical configuration.

The assumptions we have made for the above physical model are (i) the expressions of permeability, porosity, thermal conductivity, and solutal diffusivity are considered in terms of the vertical coordinate $y$; (ii) the variable viscosity is inversely proportional to a linear function of temperature, which is in the form of $\frac{1}{\mu}=\frac{1}{\mu_{\infty}}\left[1+\gamma\left(T-T_{\infty}\right)\right]$ or $\frac{1}{\mu}=a\left(T-T_{r}\right)$, (See Lai and Kulacki [1]), where $\mathrm{a}=\frac{\gamma}{\mu_{\infty}}, T_{r}=T_{\infty}-\frac{1}{\gamma}, \gamma$ is the thermal property of the fluid. (iii) the $l^{t h}$ order homogeneous chemical reaction with a constant rate $K_{R}$ between fluid and diffusing species are taken into account. The corresponding governing equations of the motion under all the above assumptions along with Boussinesq approximation are given by:

$\frac{\partial u}{\partial x}+\frac{\partial v}{\partial y}=0$

$u \frac{\partial u}{\partial x}+v \frac{\partial u}{\partial y}=\frac{1}{\rho} \frac{\partial}{\partial y}\left(\mu \frac{\partial u}{\partial y}\right)+g \beta_{T}\left(T-T_{\infty}\right)-g \beta_{C}\left(C-C_{\infty}\right)-\frac{\epsilon(y) \bar{\mu} u}{\rho k(y)}$,

$u \frac{\partial T}{\partial x}+v \frac{\partial T}{\partial y}=\frac{\partial}{\partial y}\left(\alpha(y) \frac{\partial T}{\partial y}\right)+\frac{\mu}{\rho C_{p}}\left(\frac{\partial u}{\partial y}\right)^{2}+\frac{\bar{\mu} \epsilon^{2}(y) u^{2}}{\rho C_{p} k(y)}$,

$u \frac{\partial C}{\partial x}+v \frac{\partial C}{\partial y}=\frac{\partial}{\partial y}\left(\gamma(y) \frac{\partial C}{\partial y}\right)-K_{R}\left(C-C_{\infty}\right)^{l}$, 
the variable viscosity $\theta_{r}$ is defined by (Seddeek [3])

$\theta_{r}=\frac{T_{r}-T_{\infty}}{T_{w}-T_{\infty}}=-\frac{1}{\gamma\left(T_{w}-T_{\infty}\right)}$.

Where the velocity components $u$ and $v$ are in the direction of $x$ and $y$ respectively, $\mu$ is the viscosity of the fluid, $\bar{\mu}$ is the viscosity of porous media, $\mathrm{g}$ is the acceleration due to gravity, $\mathrm{T}$ is the temperature inside the boundary layer, $T_{\infty}$ is ambient temperature, $\beta_{\mathrm{T}}$ is thermal expansion coefficient, $\beta_{\mathrm{C}}$ is coefficient of concentration expansion, $\mathrm{C}$ is foreign fluid concentration inside the boundary layer, $C_{\infty}$ is the ambient concentration, $C_{p}$ is the specific heat, $\rho$ is the free stream density, $k(y)$ is the variable permeability, $\varepsilon(y)$ is the variable porosity, $\alpha(y)$ is the variable thermal conductivity, $\gamma(y)$ is the variable solutal diffusivity, $K_{R}$ is the dimensional chemical reaction parameter, $l$ is the order of the chemical reaction, $T_{w}$ is the accelerating wall temperature.

The temperature and concentration of the physical model are maintained in two different cases: (i) Prescribed Surface Temperature and Concentration (PSTC) and (ii) Prescribed Wall Heat and Concentration Flux (PWHCF).

(i) Prescribed Surface Temperature and Concentration (PSTC) case.

For PSTC case, a known constant uniform temperature and concentration are maintained at every point of the boundary, these constants are assumed in the form of a polynomial profile of degree ' $r$ ' (temperature parameter), the corresponding boundary conditions on velocity, temperature, and concentration fields are given by:

$u=b x, v=v_{w}, T_{w}=T_{\infty}+A_{0} x^{r}, C_{w}=C_{\infty}+A_{1} x^{r} \quad$ at $y=0$,

$u=0, T=T_{\infty}, C=C_{\infty} \quad$ as $y \rightarrow \infty$,

where ' $b$ ' is accelerating surface stretching rate and $A_{0} \& A_{1}$ are arbitrary constants. By introducing the given similarity variable $\eta$ and the non-dimensional variables $f, \theta, H$. (see Acharya et al. [2]), we can solve the governing equations (1)-(4) numerically.

$\psi=(v b)^{\frac{1}{2}} x f(\eta), \quad \eta=\left(\frac{b}{v}\right)^{\frac{1}{2}} y, \quad \theta(\eta)=\frac{T-T_{\infty}}{T_{w}-T_{\infty}}, \quad H(\eta)=\frac{C-C_{\infty}}{C_{w}-C_{\infty}}$,

where $\psi(x, y)$ is the stream function, $u=\frac{\partial \psi}{\partial y}$ and $v=-\frac{\partial \psi}{\partial x}$, which implies that the velocity components are given by:

$u=b x f^{\prime}(\eta), v=-(b v)^{\frac{1}{2}} f(\eta)$.

Chandrasekhara and Namboodiri [4] introduced the following expressions for variable permeability $k(\eta)$ and variable porosity $\varepsilon(\eta)$, variable thermal conductivity $\alpha(\eta)$ and variable solutal diffusivity $\gamma(\eta)$ respectively:

$k(\eta)=k_{0}\left(1+d e^{-\eta}\right)$,

$\varepsilon(\eta)=\varepsilon_{0}\left(1+d^{*} e^{-\eta}\right)$,

$\alpha(\eta)=\alpha_{0}\left\{\varepsilon_{0}\left(1+d^{*} e^{-\eta}\right)+\sigma^{*}\left(1-\varepsilon_{0}\left(1+d^{*} e^{-\eta}\right)\right)\right\}$,

$\gamma(\eta)=\gamma_{0}\left\{\varepsilon_{0}\left(1+d^{*} e^{-\eta}\right)+\gamma^{*}\left(1-\varepsilon_{0}\left(1+d^{*} e^{-\eta}\right)\right)\right\}$, 
where $k_{0}, \varepsilon_{0}, \alpha_{0}$ and $\gamma_{0}$ are the permeability, porosity, thermal conductivity, and solutal diffusivity at the edge of the boundary layer, respectively and $d=3.0 \& d^{*}=1.5$ are treated as constants for variable permeability and porosity, respectively, $\sigma^{*}$ is the ratio of thermal conductivity of the solid to the conductivity of the fluid and $\gamma^{*}$ is the ratio of solutal diffusivity of the solid to the diffusivity of the fluid.

The transformed non-dimensional coupled non-linear ordinary differential equations can be obtained by substituting Eqs. (8) \& (9) in Eqs. (2) - (4) using Eqs. (10) - (13) as follows:

$$
\begin{gathered}
f^{\prime \prime \prime}-\left(\frac{1}{\theta-\theta_{r}}\right) \theta^{\prime} f^{\prime \prime}-\left(\frac{\theta}{\theta_{r}}-1\right)\left(f f^{\prime \prime}-f^{\prime 2}\right)+\alpha^{*} \epsilon_{0} \sigma\left(\frac{1+d^{*} e^{-\eta}}{1+d e^{-\eta}}\right)\left(\frac{\theta}{\theta_{r}}-1\right) f^{\prime}= \\
\left(\frac{\theta}{\theta_{r}}-1\right)(G s \theta-G c H), \\
\begin{array}{r}
\theta^{\prime \prime}\left(\epsilon_{0}+\sigma^{*}\left(1-\epsilon_{0}\right)+\epsilon_{0} d^{*} e^{-\eta}\left(1-\sigma^{*}\right)\right)=r \operatorname{Pr} f^{\prime} \theta-\operatorname{Pr} f \theta^{\prime}-d^{*} e^{-\eta} \epsilon_{0}\left(\sigma^{*}-1\right) \theta^{\prime} \\
-E \operatorname{Pr} f^{\prime \prime 2}-E \sigma \operatorname{Pr} \epsilon_{0}^{2}\left[\frac{\left(1+d^{*} e^{-\eta}\right)^{2}}{1+d e^{-\eta}}\right] f^{\prime 2}, \\
H^{\prime \prime}\left(\epsilon_{0}+\gamma^{*}\left(1-\epsilon_{0}\right)+\epsilon_{0} d^{*} e^{-\eta}\left(1-\gamma^{*}\right)\right)=r S c f^{\prime} H-S c f H^{\prime}+k_{r} H_{-}^{l} \\
d^{*} e^{-\eta} \epsilon_{0}\left(\gamma^{*}-1\right) H^{l},
\end{array}
\end{gathered}
$$

the transformed boundary conditions in the non-dimensional form are:

$$
\begin{aligned}
& f(0)=-\frac{v_{w}}{(b v)^{\frac{1}{2}}}=-m, \quad f^{\prime}(0)=1, \quad \theta=1, \quad H=1 \quad \text { at } y=0, \\
& f^{\prime}=0, \quad \theta=0, H=0 \quad \text { as } y \rightarrow \infty .
\end{aligned}
$$

\section{(ii) Prescribed Wall Heat and Concentration Flux (PWHCF) case.}

For the PWHCF case, both temperature and concentration vary with respect to the coordinate axis $y$ and the corresponding boundary conditions on velocity, temperature, and concentration fields are given by:

$u=b x, v=v_{w},-k \frac{\partial T}{\partial y}=q_{w} E_{0} x^{s},-D \frac{\partial C}{\partial y}=m_{w} E_{1} x^{s} \quad$ at $y=0$,

$u=0, T=T_{\infty}, C=C_{\infty} \quad$ as $y \rightarrow \infty$,

where $E_{0}, E_{1}$ are choosing constants, ' $s$ ' is the heat flux parameter. Similarly, by introducing the similarity variable $\eta$ and the non-dimensional variables $f, g, h$. (see Acharya et al. [2]), we can transform the Eqs (1)-(4).

$\psi=(v b)^{\frac{1}{2}} x f(\eta), \quad \eta=\left(\frac{b}{v}\right)^{\frac{1}{2}} y, T-T_{\infty}=\frac{E_{0} x^{s}}{k}\left(\frac{v}{a}\right)^{\frac{1}{2}} g(\eta), C-C_{\infty}=\frac{E_{1} x^{s}}{D}\left(\frac{v}{a}\right)^{\frac{1}{2}} h(\eta)$,

The transformed non-dimensional coupled non-linear ordinary differential equations can be obtained by substituting Eqs. (9) \& (21) in Eqs. (2) - (4) using Eqs. (10) - (13) as follows:

$$
\begin{aligned}
f^{\prime \prime \prime}-\left(\frac{1}{g-g_{r}}\right) g^{\prime} f^{\prime \prime}-\left(\frac{g}{g_{r}}-1\right)\left(f f^{\prime \prime}-f^{\prime 2}\right)+\alpha^{*} \epsilon_{0} \sigma\left(\frac{1+d^{*} e^{-\eta}}{1+d e^{-\eta}}\right)\left(\frac{g}{g_{r}}-1\right) f^{\prime}= \\
\left(\frac{g}{g_{r}}-1\right)(G s g-G c h), \\
g^{\prime \prime}\left(\epsilon_{0}+\sigma^{*}\left(1-\epsilon_{0}\right)+\epsilon_{0} d^{*} e^{-\eta}\left(1-\sigma^{*}\right)\right)=s \operatorname{Pr}^{\prime} g-\operatorname{Pr} f g^{\prime}-d^{*} e^{-\eta} \epsilon_{0}\left(\sigma^{*}-1\right) g^{\prime} \\
-E \operatorname{Pr} f^{\prime \prime 2}-E \sigma \operatorname{Pr} \epsilon_{0}{ }^{2}\left[\frac{\left(1+d^{*} e^{-\eta}\right)^{2}}{1+d e^{-\eta}}\right] f^{\prime 2},
\end{aligned}
$$




$$
\begin{gathered}
h^{\prime \prime}\left(\epsilon_{0}+\gamma^{*}\left(1-\epsilon_{0}\right)+\epsilon_{0} d^{*} e^{-\eta}\left(1-\gamma^{*}\right)\right)=s S c f^{\prime} h-S c f H^{\prime}+k_{r} h^{l}- \\
d^{*} e^{-\eta} \epsilon_{0}\left(\gamma^{*}-1\right) h^{l},
\end{gathered}
$$

the transformed boundary conditions in non-dimensional form are:

$$
\begin{aligned}
& f(0)=-\frac{v_{w}}{(b v)^{\frac{1}{2}}}=-m, \quad f^{\prime}(0)=1, g=-1, h=-1 \quad \text { at } y=0, \\
& f^{\prime}=0, \theta=0, H=0 \quad \text { as } y \rightarrow \infty .
\end{aligned}
$$

Where all the dimensionless parameters present in the above equations are defined in nomenclature, and the prime represents differentiation concerning to $\eta$.

\subsection{Method of solution.}

Applying the known analytical techniques makes it very difficult to obtain the solution of the system of coupled highly nonlinear differential equations with variable coefficients. So, transform the higher-order coupled nonlinear ODE's into a system of first-order ODE's to overcome the difficulty that occurred in the analytical procedure. The transformed first-order ODE's can be solved numerically by the shooting technique, which combines the second-order Newton-Raphson method and Runge-Kutta-Fehlberg explicit method. For the PSTC case, we determined the numerical iterative solution for the functions of $f(\eta), \theta(\eta) \& H(\eta)$ by solving the Eqs. (14) - (16) along with the boundary conditions (17) \& (18) and similarly for the functions of $f(\eta), g(\eta) \& h(\eta)$, solve the Eqs. (22) - (24) along with the boundary conditions (25) \& (26) in PWHCF case. Using MatLab software, we developed a program to compute the numerical results, and in which we maintained the numerical values are less than the prescribed accuracy of $10^{-6}$.

\section{Results and Discussion}

The importance of this work is to understand the variations of fluid properties like viscosity, permeability, porosity, thermal conductivity, and solutal diffusivity over an accelerating surface under the influence of chemical reactions. The numerical results are carried out to discuss the variation of velocity or momentum of the fluid flow, temperature difference, and solutal changes are depicted from Figures 2-15 for the non-dimensional parameters like a ratio of solutal diffusivity, a ratio of thermal conductivity, viscosity parameter, porous parameter, Eckert number, Prandtl number, chemical reaction parameter, temperature and mass buoyancy parameters involved in the physical problem. In the entire study, we employ the fixed values of nondimensional parameters $\operatorname{Pr}=2.0, E=0.6, G s=0.1, G c=0.1, \theta_{r}=5.0, g_{r}=3.0, S c=$ 1.0, $r=1, s=1, m=0.5, \epsilon_{0}=0.1, \quad d=3.0, d^{*}=0.5, \quad \sigma=2.0, \alpha^{*}=1.0, \sigma^{*}=3.0$, $\gamma^{*}=2.0, K_{r}=0.2$ and $l=2$ except the values varied as displayed in respective figures. Also, it is observed that in the absence of chemical reaction and for a constant viscosity over a nonaccelerating vertical plate, i.e., $b=0.0$ (stretching rate), the numerical results are obtained and compared with the published work of Nalinakshi et al. [6].

\subsection{Effect of viscosity parameter $\left(\theta_{r}\right)$.}

Figures 2-4 demonstrated the variations of dimensionless velocity, temperature, and concentration for different values of viscosity parameter $\theta_{r}$, respectively. The velocity profile decreases for the enhancement of the viscosity parameter $\theta_{r}$ because higher viscosity reduces the 
flow rate of the fluid, it can be gleaned from Figure 2. But a reverse trend can be observed from Figure 3 in the case of the temperature profile. This is because, with an increase of temperature (i.e., for decrement of temperature difference $\Delta T$, viscosity parameter will increase), the fluid becomes lighter, which results in the heat transfer being more in the PWHCF case than the PSTC case. Similarly, the variations of dimensionless concentration for different values of viscosity parameter $\theta_{r}$ are shown in Figure 4. Due to the indirect involvement (i.e., through momentum and energy equations) of the viscosity parameter $\theta_{r}$ in the species equation, the effect of the viscosity parameter $\theta_{r}$ is very less in concentration profile compared to velocity and temperature profiles.

\subsection{Effect of a porous parameter $(\sigma)$.}

Figures 5-7 display that the variations of dimensionless velocity, temperature, and concentration for different values of porous parameter $\sigma$. The porous parameter changes with respect to the stretching rate of the accelerating plate or viscosity of the fluid or permeability at the edge of the boundary layer. So, an increase of porous parameters will decrease the flow velocity of the fluid, which is depicted in Figure 5. Quite the opposite trend can be gleaned from Figures 6 and 7 with temperature and concentration profiles for various values of the porous parameter $\sigma$, i.e., the dimensionless temperature and concentration increase in both the cases of PSTC and PWHCF. Our results well coincide with the previous work of Seddeek [5] for $\sigma=0$ (i.e., in the absence of permeability of porous medium).

\subsection{Effect of ratio of thermal conductivity $\left(\sigma^{*}\right)$.}

Figures 8 and 9 represent the behavior of the velocity and temperature profiles for different values of the ratio of thermal conductivity $\sigma^{*}$, respectively. It is noticed that with the increase of the ratio of thermal conductivity of solid to the conductivity of the fluid $\sigma^{*}$ both velocity and temperature distributions increase exponentially; this is because with higher values of $\sigma^{*}$ leads to enhancing the thermal boundary layer with higher thermal conductivity. The effect is more near the accelerating plate and is less far away from the accelerating surface.

\subsection{Effect of ratio of solutal diffusivity $\left(\gamma^{*}\right)$.}

We can observe the effect of the ratio of solutal diffusivity of solid to the diffusivity of fluid $\gamma^{*}$ on dimensionless concentration profile from Figure 10. The dimensionless concentration increases with an increase in the ratio of solutal diffusivity $\gamma^{*}$, this is due to the fact that the solutal boundary layer overshoots with higher values of $\gamma^{*}$.

\subsection{Effect of chemical reaction parameter $\left(K_{r}\right)$.}

Figure 11 indicates the behavior of the concentration profiles for different values of the chemical reaction parameter $K_{r}$ and it is observed that the fluid concentration gradients reduce for higher values of chemical reaction parameter and higher order of chemical reaction in both the cases of PSTC and PWHCF, which is since with the higher values of $K_{r}$ increases the thickness of the solutal boundary layer, which reduces the concentration species diffusion and decelerates the mass diffusion. 


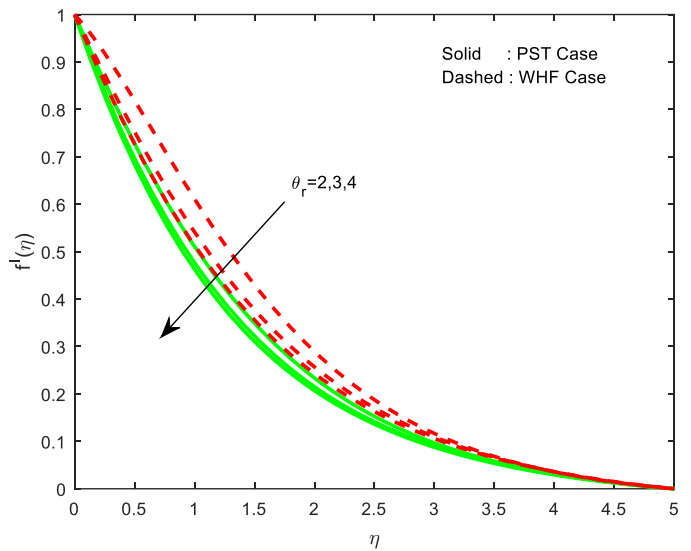

Figure 2. Velocity variations of viscosity parameter.

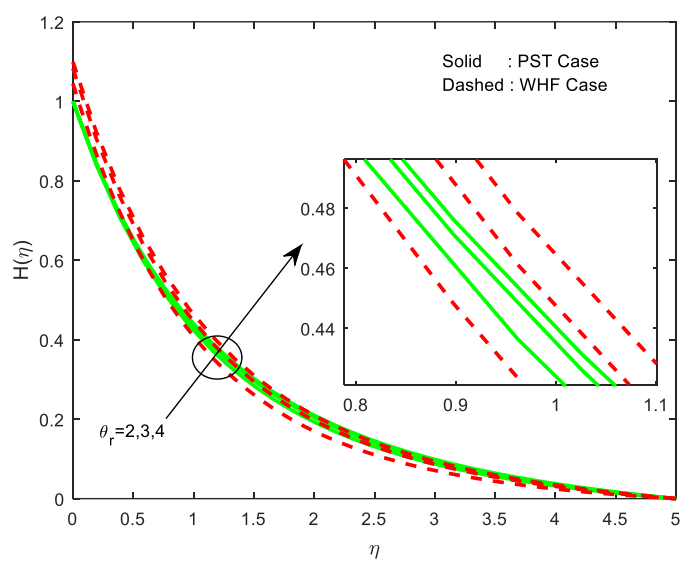

Figure 4. Concentration variations of viscosity parameter.

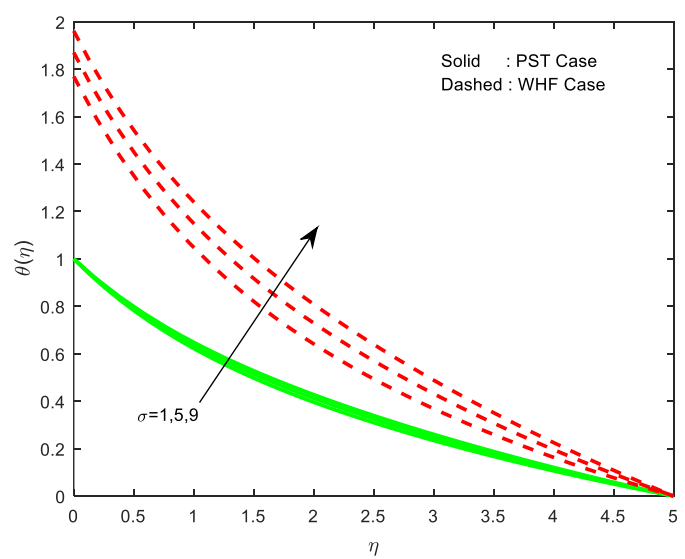

Figure 6. Temperature variations of a local porous parameter.

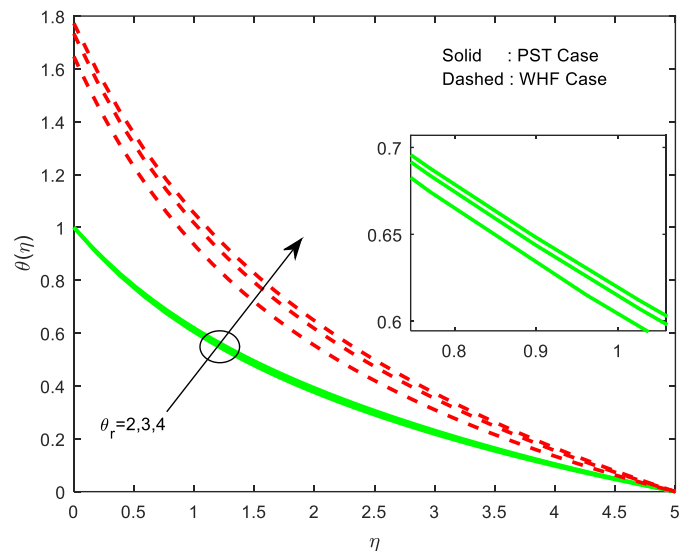

Figure 3. Temperature variations of viscosity parameter.

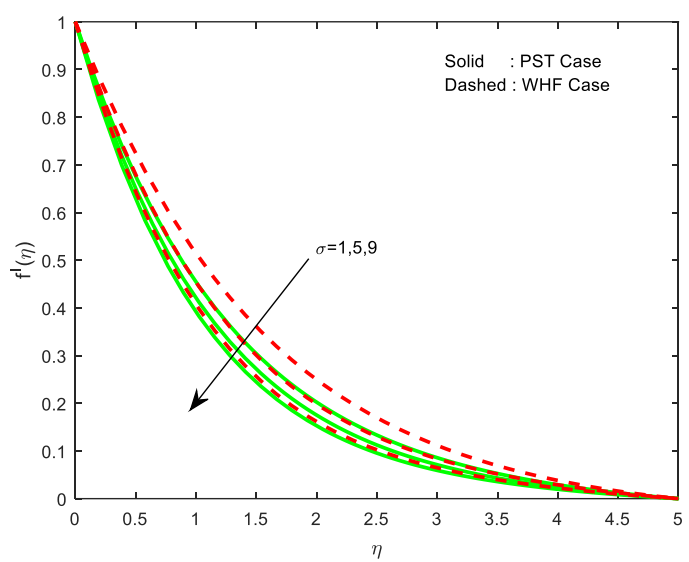

Figure 5. Velocity variations of local porous parameter.

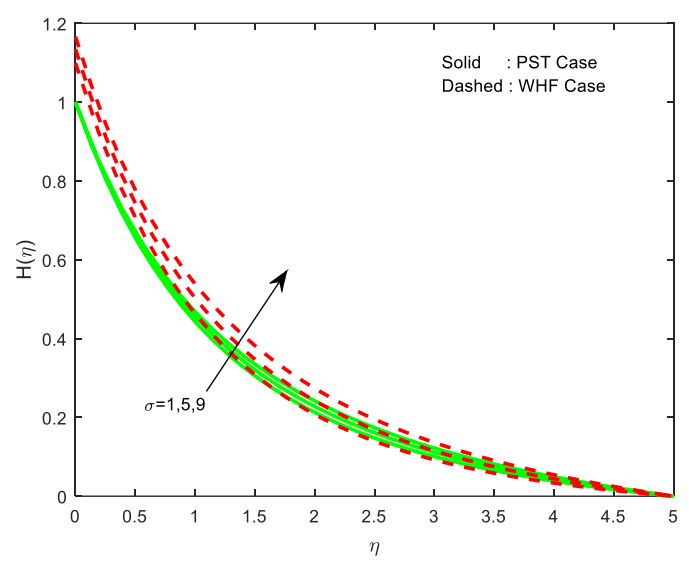

Figure 7. Concentration variations of local porous parameter.

\subsection{Effect of Prandtl number(Pr).}

The variations of Prandtl numbers in terms of velocity, temperature, and concentration are shown in Figures 12-14. The changes of the Prandtl number are due to an increment of fluid viscosity or a decrement of thermal diffusivity. The increment of fluid viscosity reduces the fluid's flow velocity and temperature, which can be observed in Figures 12 and 13. Whereas in the case of concentration profile, the opposite behavior can be gleaned from Figure 14, i.e., the dimensionless concentration gradually increases with the higher values of the Prandtl number. But 
the effect of Prandtl number is very less on concentration profile for both PSTC and PWHCF compared to that of velocity and temperature profiles because the equation of species is not directly affected by the Prandtl number affected through the energy equation.

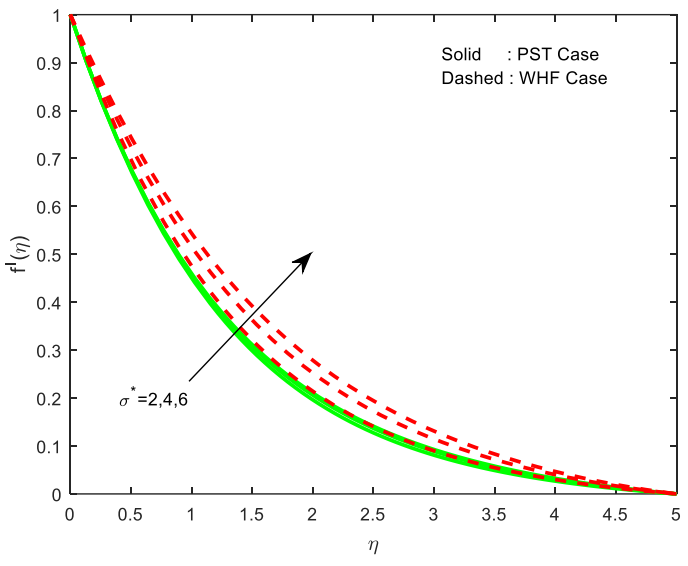

Figure 8. Velocity variations of ratio of thermal conductivities.

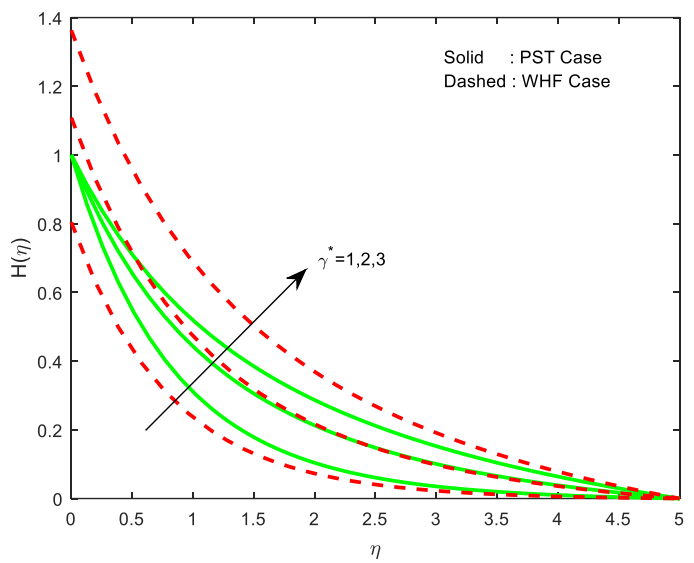

Figure 10. Concentration variations of ratio of solutal diffusivities

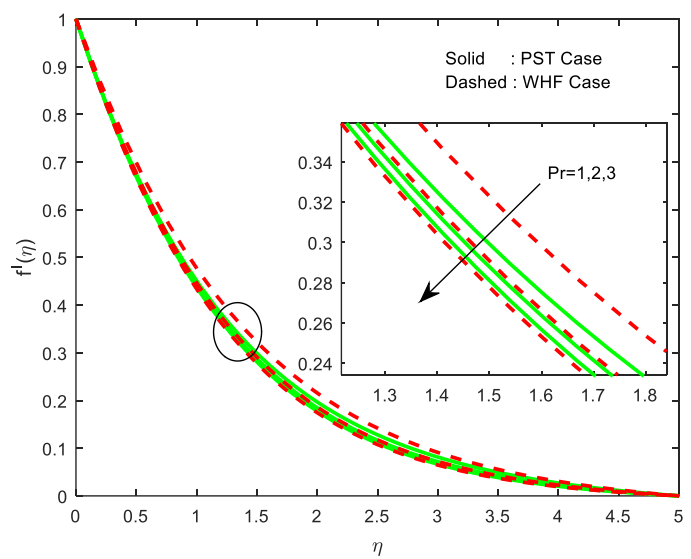

Figure 12. Velocity variations of Prandtl number.

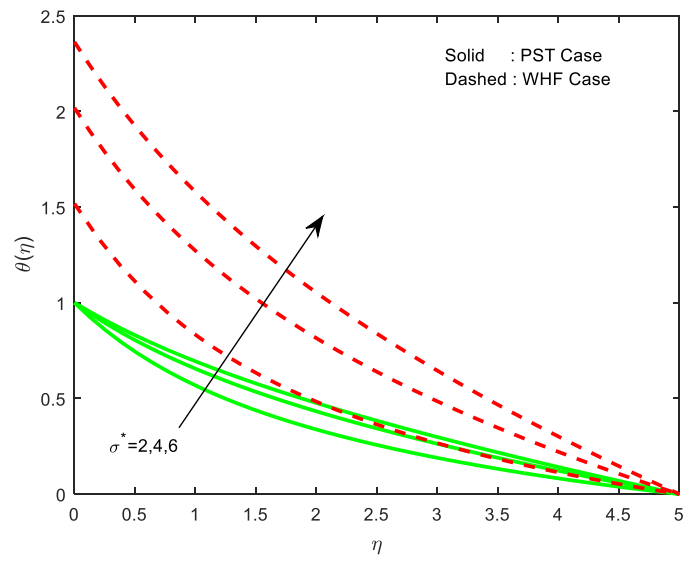

Figure 9. Temperature variations of ratio of thermal conductivities.

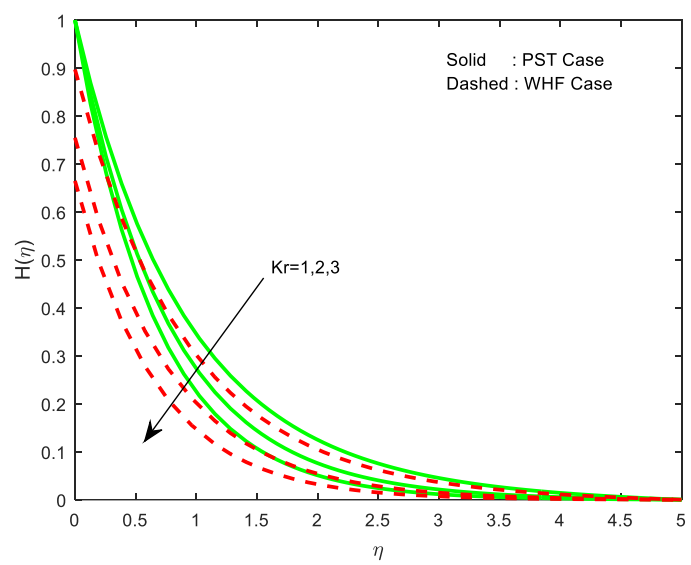

Figure 11. Concentration variations of chemical reaction parameter.

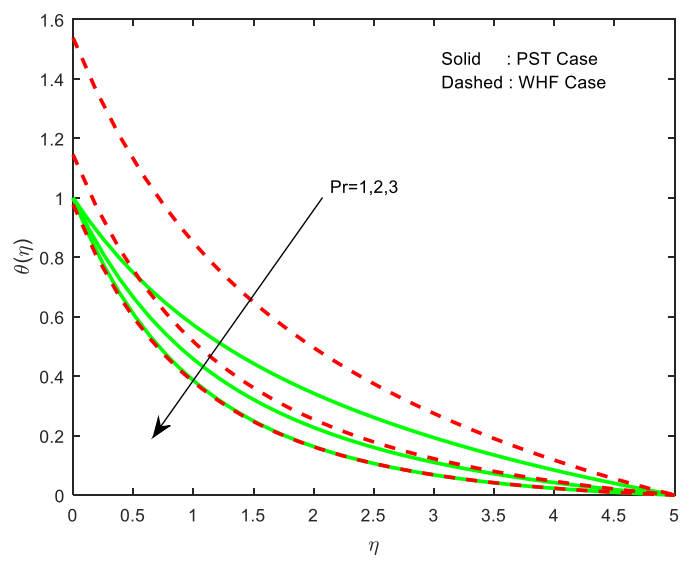

Figure 13. Temperature variations of Prandtl number.

\subsection{Effect of Eckert number(E).}

Figure 15 illustrates the behavior of the non-dimensional temperature profile for different values of the Eckert number $E$. It is noticed that the enhancement of Eckert number $E$ accelerates 
the dimensionless temperature gradient in both the cases of PSTC and PWHCF, which is due to the stretching enhancement rate of accelerating surface results increase the kinetic energy of the fluid flow or which is due to decrease in the temperature difference $\Delta T$ in the physical model.

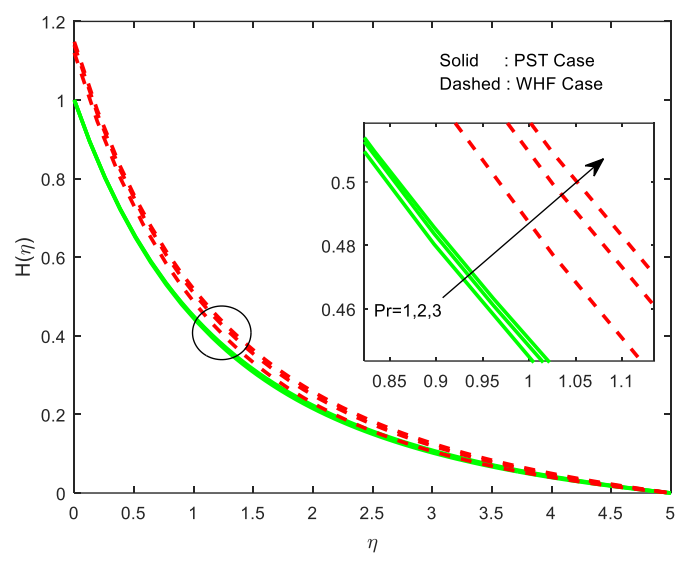

Figure 14. Concentration variations of Prandtl number.

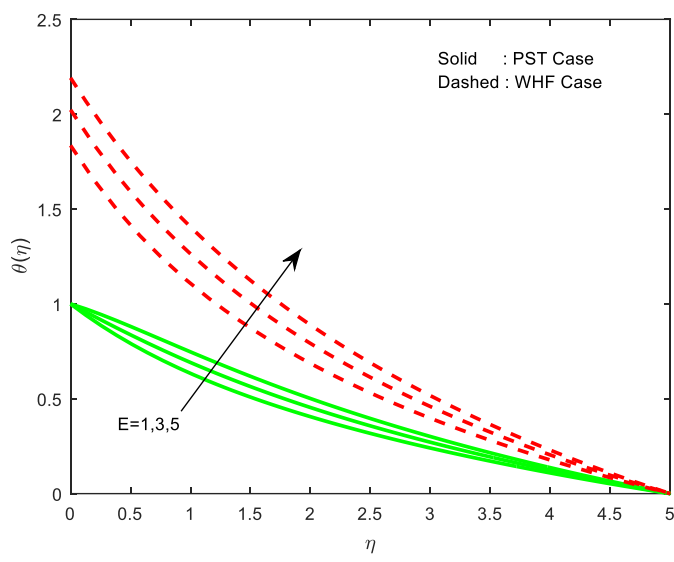

Figure 15. Temperature variations of Eckert number.

\section{Conclusions}

This study investigated the effect of chemical reactions on double-diffusive (heat and mass) mixed convection flow with variable fluid properties like variable viscosity, porosity, permeability, thermal conductivity, and solutal diffusivity porous medium over an accelerating surface. The computed numerical results are presented to analyze the flow, mass, and heat transfer characteristics of the fluid under the influence of various physical parameters; the following conclusions can be drawn from the present study:

- The increase of viscosity parameter $\left(\theta_{r}\right)$ reduces the velocity of the fluid flow, whereas it enhances the temperature and concentration profiles. The quite same behavior can be observed for a porous parameter $(\sigma)$.

- For the higher values of the ratio of thermal conductivities $\left(\sigma^{*}\right)$ the boundary layer flow attains high velocity and temperature. Similarly, an increase in the ratio of solutal diffusivities $\left(\gamma^{*}\right)$ enhances the concentration boundary layer.

- It is observed that the dimensionless concentration decreases with an increase of chemical reaction parameter $\left(K_{r}\right)$.

- Higher values of Prandtl number $(P r)$ decrease the velocity and temperature profile, whereas the trend is reversed in the case of concentration profile. Similarly, an increase in the Eckert number $(E)$ enhances the temperature profile.

\section{Funding}

This research received no external funding.

\section{Acknowledgments}

The authors are thankful to the research center, Department of Mathematics, M. S. Ramaiah Institute of Technology, Bangalore, affiliated to VTU for their valuable support to our research work. 


\section{Conflicts of Interest}

The authors declare no conflict of interest.

\section{References}

1. Lai, F.C.; Kulacki, F.A. The effect of variable viscosity on convective heat transfer along a vertical surface in a saturated porous medium. Int. J. Heat Mass Transfer 1990, 33, 1028-1031, https://doi.org/10.1016/00179310(90)90084-8.

2. Acharya, M.; Singh, L.P.; Dash, G.C. Heat and mass transfer over an accelerating surface with a heat source in presence of suction and blowing. International Journal of Engineering Science 1999, 37, 189-211, https://doi.org/10.1016/S0020-7225(98)00064-0.

3. Seddeek, M.A. The effect of variable viscosity on hydromagnetic flow and heat transfer past a continuously moving porous boundary with radiation. International Communications in Heat and Mass Transfer 2000, 27, 1037-1046, https://doi.org/10.1016/S0735-1933(00)00183-4.

4. Chandrasekhara, B.C.; Namboodiri, P.M.S. Influence of variable permeability on combined vertical surfaces in a porous medium. International Journal of Heat Mass Transfer 1985, 28, 199-206, https://doi.org/10.1016/00179310(85)90022-5.

5. Seddeek, M.A. Thermal-diffusion and diffusion-thermo effects on mixed free-forced convective flow and mass transfer over an accelerating surface with a heat source in the presence of suction and blowing in the case of variable viscosity. Acta Mechanica 2004, 172, 83-94, https://doi.org/10.1007/s00707-004-0139-5.

6. Nalinakshi, N.; Dinesh, P. A.; Chandrashekhar, D. V. Numerical Study of Double Diffusive mixed convection with variable fluid properties. International Journal of Engineering Research and Technology 2013, 2, 131-139, https://www.ijert.org/research/numerical-study-of-double-diffusive-mixed-convection-with-variable-fluidproperties-IJERTV2IS90120.pdf.

7. Girinath Reddy, M.; Dinesh, P.A.; Sandeep, N. Effects of variable viscosity and porosity of fluid, Soret and Dufour mixed double-diffusive convective flow over an accelerating surface. IOP Conference Series: Materials Science and Engineering 2017, 263, 062012, https://doi.org/10.1088/1757-899X/263/6/062012.

8. Girinath Reddy, M.; Dinesh, P.A. Double diffusive convection and internal heat generation with Soret and Dufour effects over an accelerating surface with variable viscosity and permeability. Advances in Physics Theories and Applications 2018, 69, 7-25, https://www.iiste.org/Journals/index.php/APTA/article/view/40558/41714.

9. Krishna Prasad, D.V.; Krishna Chaitanya, G.S.; Srinivasa Raju, R. Double diffusive effects on mixed convection Casson fluid flow past a wavy inclined plate in presence of Darcian porous medium, Results in Engineering 2019, 3, 1-15, https://doi.org/10.1016/j.rineng.2019.100019.

10. Manjunatha, S.; Kuttan, A.; Jayanthi, S.; Chamkha, A.; Gireesha, B.J. Heat transfer enhancement in the boundary layer flow of hybrid nanofluids due to variable viscosity and natural convection. Heliyon 2019, 5, 1-16, https://doi.org/10.1016/j.heliyon.2019.e01469.

11. Shilpa, B. V.; Chandrashekar, D. V.; Dinesh, P. A.; Eswara, A. T. Analytical approach for mixed convective flow in presence of Casson fluid in a Porous channel. Advances in Fluid Dynamics, Lecture Notes in Mechanical Engineering 2020, 939-952, https://doi.org/10.1007/978-981-15-4308-1_71.

12. Sravan Kumar, T.; Dinesh, P. A.; Makinde, O. D. Impact of Lorentz force and viscous dissipation on unsteady nanofluid convection flow over an exponentially moving vertical plate. Mathematical Models and Computer Simulations 2020, 12, 631-646, https://doi.org/10.1134/S2070048220040110.

13. Khan, S.U.; Vaidya, H.; Chammam, W.; Musmar, S.A.; Prasad, K.V.; Tlili, I. Triple Diffusive Unsteady Flow of Eyring-Powell Nanofluid Over a Periodically Accelerated Surface With Variable Thermal Features. Front. Phys. 2020, 8, 246, https://doi.org/10.3389/fphy.2020.00246.

14. Ayegbusi Florence; Onwubuoya Cletus; Bidemi Falodun. The unsteady problem of magnetohydrodynamic heat plus mass transfer convective flow over a moveable plate with effects of thermophoresis and thermal radiation, Heat Transfer 2020, 49, 3593-3612, https://doi.org/10.1002/htj.21790.

15. Ramachandramurthy, V.; Aruna, A. S.; Kavitha, N. Benard-Taylor convection in temperature-dependent variable viscosity Newtonian liquids with an internal heat source. Int. J. Appl. Comput. Math. 2020, 6, https://doi.org/10.1007/s40819-020-0781-1. 
16. Basavaraj, M. S.; Girinath Reddy, M.; Aruna, A.S.; Dinesh P.A. A nonlinear mixed convective oscillatory flow over a semi-infinite vertical plate through a porous medium under uniform magnetic field. Int. Journal of Advanced Research 2020, 8, 308-321, https://dx.doi.org/10.21474/IJAR01/11100.

17. Nima, N.I.; Ferdows, M.; Adesanya, S.O. Double-diffusivity heat generation effects on bioconvection process embedded in a vertical porous surface with variable fluid properties. $J$ Therm Anal Calorim 2020, https://doi.org/10.1007/s10973-020-09822-5.

18. Anantha Kumar Kempannagari; Ramoorthy Reddy Buruju; Sandeep Naramgari; Sugunamma Vangala. Effect of Joule heating on MHD non-Newtonian fluid flow past an exponentially stretching curved surface. Heat transfer 2020, 49, 3575-3592, https://doi.org/10.1002/htj.21789.

19. Wahab, H.A.; Zeb, H.; Bhatti, S.; Gulistan, M.; Kadry, S.; Nam, Y. Numerical Study for the Effects of Temperature-Dependent Viscosity Flow of Non-Newtonian Fluid with Double Stratification. Appl. Sci. 2020, 10, 708, https://doi.org/10.3390/app10020708.

20. Nalinakshi, N.; Dinesh, P. A. Thermo-diffusion and diffusion-thermo effects for a Forchheimer model with MHD over a vertical heated plate. Advances in Fluid Dynamics(LNME) 2020, 343-361, https://doi.org/10.1007/978-981-15-4308-1_27.

21. Sami, Ullah, Khan; Sabir, Ali, Shehzad. Electrical MHD Carreau nanofluid over a porous oscillatory stretching surface with variable thermal conductivity: Applications of thermal extrusion system. Physica A: Statistical Mechanics and its Applications 2020, 550, 0378-4371, https://doi.org/10.1016/j.physa.2020.124132.

22. Gangadhar, K.; Kannan, T.; DasaradhaRamaiah, K.; Sakthivel, G. Boundary layer flow of nanofluids to analyse the heat absorption/generation over a stretching sheet with variable suction/injection in the presence of viscous dissipation. International Journal of Ambient Energy 2020, 41, 969-980, https://doi.org/10.1080/01430750.2018.1501738.

23. Uma, M.; Dinesh, P.A.; Girinath Reddy, M.; Sreevallabha Reddy, A. Combined effects of Forchheimer, Soret and Dufour on MHD mixed convective dusty viscoelastic Couette flow in an irregular channel. Multidiscipline Modeling in Materials and Structures 2021, 17, 49-64, https://doi.org/10.1108/MMMS-12-2019-0233.

24. Apelblat, A., Mass transfer with a chemical reaction of the first order: analytical solutions. Chem. Eng. J. 1980, 19, 19-37, https://doi.org/10.1016/0300-9467(80)85074-X.

25. Afify, A.A.; Ahmed, A. Free convective flow and mass transfer over a stretching sheet with chemical reaction. Heat and Mass Transfer 2004, 40, 495-500, https://doi.org/10.1007/s00231-003-0486-0.

26. Rashad, A.M.; El-Kabeir, S.M.M. Heat and mass transfer in transient flow by mixed convection boundary layer over a stretching sheet embedded in a porous medium with chemically reactive species. J. Porous Media 2010, 13, 75-85, http://dx.doi.org/10.1615/JPorMedia.v13.i1.70.

27. Shreeedhar, G; Rama Bhupal Reddy, B. Chemical reaction effect on unsteady MHD flow past an infinite vertical porous plate in the presence of heat absorption. International Journal of Advanced Research in Engineering and Technology 2019, 10, 95-103, https://doi.org/10.34218/IJARET.10.1.2019.010.

28. Musa, Antidius, Mjankwi; Verdiana, Grace, Masanja; Eunice, W., Mureithi; Makungu, Ng'oga, James. Unsteady MHD Flow of Nanofluid with Variable Properties over a Stretching Sheet in the Presence of Thermal Radiation and Chemical Reaction. International Journal of Mathematics and Mathematical Sciences 2019, 1-14, https://doi.org/10.1155/2019/7392459.

29. Prasad, P.D.; Saleem, S.; Varma, S.V.K. Three Dimensional Slip Flow of a Chemically Reacting Casson Fluid Flowing over a Porous Slender Sheet with a Non-Uniform Heat Source or Sink. J. Korean Phys. Soc. 2019, 74, 855-864, https://doi.org/10.3938/jkps.74.855.

30. Dinesh, P.A.; Vasudevamurthy, A. S.; Uma, M. Effects of Forchheimer, MHD and radiation adsorption for chemically reacting unsteady dusty viscoelastic fluid Couette flow in an irregular channel. Advances in Fluid Dynamics (LNME) 2020, 999-1012, https://doi.org/10.1007/978-981-15-4308-1_77.

31. Shafiq, A.; Rasool, G.; Khalique, C.M.; Aslam, S. Second Grade Bioconvective Nanofluid Flow with Buoyancy Effect and Chemical Reaction. Symmetry 2020, 12, 621, https://doi.org/10.3390/sym12040621.

32. Sehra, Haq, S.U.; Shah, S.I.A. Convection heat mass transfer and MHD flow over a vertical plate with chemical reaction, arbitrary shear stress and exponential heating. Sci. Rep. 2021, 11, 4265, https://doi.org/10.1038/s41598021-81615-8. 